\title{
Embedding theorems for composition of homotopy and projection operators
}

\author{
Jinling Niu', Shusen Ding ${ }^{2}$ and Yuming Xing ${ }^{1 *}$
}

\section{"Correspondence:}

xyuming@hit.edu.cn

'Department of Mathematics,

Harbin Institute of Technology,

Harbin, 150001, China

Full list of author information is

available at the end of the article
2015 Niu et al. This article is distributed under the terms of the Creative Commons Attribution 4.0 International License (http://creativecommons.org/licenses/by/4.0/), which permits unrestricted use, distribution, and reproduction in any medium, provided you give appropriate credit to the original author(s) and the source, provide a link to the Creative Commons license, and indicate if changes were made.

\begin{abstract}
We prove both local and global embedding theorems with $L^{\varphi}$-norms for the composition of the homotopy operator and projection operator applied to differential forms. We also establish some $L^{\varphi}$-norm inequalities for certain compositions of the related operators.
\end{abstract}

MSC: Primary 35J60; secondary 35B45; 30C65; 47J05; 46E35

Keywords: embedding inequalities; differential forms; homotopy operators; projection operators

\section{Introduction}

The homotopy operator $T$ and projection operator $H$ defined on differential forms are two critical operators which have been very well studied and used in recent years, see [1-7]. In many situations, we need to deal with the composition $T \circ H$ of the homotopy operator $T$ and the projection operator $H$. For example, when we consider the decomposition of $H(u)$, we have to face the composition $T \circ H$. The study of the composition $T \circ H$ of homotopy and projection operators was initiated by Ding and Liu in 2009 in [5] and [6], respectively, where they investigated singular integrals of this composite operator and established some $L^{p}$ inequalities for the composite operator $T \circ H$ with singular factors. Later, in 2011, Bi and Ding proved some $L^{\varphi}$-estimates for this composite operator $T \circ H$ in [7], where $\varphi$ satisfies the $G(p, q, C)$ condition. The purpose of this paper is to establish the $L^{\varphi}$-embedding theorems for the composition $T \circ H$ applied to differential forms, here $\varphi$ satisfies the $N G(p, q)$ condition. If we choose $\varphi(t)=t^{p}$, the $L^{\varphi}$-norm inequalities reduce to $L^{p}$-norm inequalities. Our main $L^{\varphi}$-embedding inequality for the composite operator can be simply stated as

$$
\left\|T(H(u))-(T(H(u)))_{\Omega}\right\|_{W^{1, \varphi}(\Omega)} \leq C\|u\|_{L^{\varphi}(\Omega)},
$$

where $\Omega$ is any bounded domain in $\mathbb{R}^{n}, n \geq 2, \varphi:[0, \infty) \rightarrow[0, \infty)$ with $\varphi(0)=0$ is a Young function satisfying certain conditions described later, and $C$ is a constant independent of the differential form $u$. In order to establish the above main $L^{\varphi}$-embedding inequality, we also prove the Poincaré inequality and some inequalities with $L^{\varphi}$-norm for the related compositions of operators. 
We keep using the traditional notations throughout this paper. Let $B$ and $\sigma B$ be the balls with the same center and $\operatorname{diam}(\sigma B)=\sigma \operatorname{diam}(B)$. Let $|E|$ be the $n$-dimensional Lebesgue measure of a set $E \subseteq \mathbb{R}^{n}$. In this paper, we treat a ball same as a cube and use $u_{B}=\frac{1}{|B|} \int_{B} u d x$ to denote the average of a function $u$. Let $\wedge^{l}=\wedge^{l}\left(\mathbb{R}^{n}\right)$ be the set of all $l$-forms in $\mathbb{R}^{n}$, $D^{\prime}\left(\Omega, \wedge^{l}\right)$ be the space of all differential $l$-forms in $\Omega$, and $L^{p}\left(\Omega, \wedge^{l}\right)$ be the $l$-forms $u(x)=$ $\sum_{I} u_{I}(x) d x_{I}$ in $\Omega$ satisfying $\int_{\Omega}\left|u_{I}\right|^{p}<\infty$ for all ordered $l$-tuples $I, l=1,2, \ldots, n$. We denote the exterior derivative by $d$ and the Hodge star operator by $\star$.

The definition of the operator $K_{y}$ with the case $y=0$ and its generalized version can be found in $[8,9]$. To each $y \in \Omega$ there corresponds a linear operator $K_{y}: C^{\infty}\left(\Omega, \wedge^{l}\right) \rightarrow$ $C^{\infty}\left(\Omega, \wedge^{l-1}\right)$ defined by $\left(K_{y} \omega\right)\left(x ; \xi_{1}, \ldots, \xi_{l-1}\right)=\int_{0}^{1} t^{l-1} \omega\left(t x+y-t y ; x-y, \xi_{1}, \ldots, \xi_{l-1}\right) d t$ and the decomposition $\omega=d\left(K_{y} \omega\right)+K_{y}(d \omega)$. A homotopy operator $T: C^{\infty}\left(\Omega, \wedge^{l}\right) \rightarrow C^{\infty}\left(\Omega, \wedge^{l-1}\right)$ is defined by averaging $K_{y}$ over all points $y \in \Omega: T \omega=\int_{\Omega} \phi(y) K_{y} \omega d y$, where $\phi \in C_{0}^{\infty}(\Omega)$ is normalized so that $\int \phi(y) d y=1$. For each differential form $u$, we have the decomposition

$$
u=d(T u)+T(d u)
$$

and

$$
\|\nabla(T u)\|_{p, B} \leq C|B|\|u\|_{p, B} \quad \text { and } \quad\|T u\|_{p, B} \leq C|B| \operatorname{diam}(B)\|u\|_{p, B}
$$

From [10], p.16, we know that any open subset $\Omega$ in $\mathbb{R}^{n}$ is the union of a sequence of cubes $Q_{k}$, whose sides are parallel to the axes, whose interiors are mutually disjoint, and whose diameters are approximately proportional to their distances from $F$, where $F$ is the complement of $\Omega$ in $\mathbb{R}^{n}$. Specifically, (i) $\Omega=\bigcup_{k=1}^{\infty} Q_{k}$, (ii) $Q_{j}^{0} \cap Q_{k}^{0}=\phi$ if $j \neq k$, (iii) there exist two constants $c_{1}, c_{2}>0$ (we can take $c_{1}=1$, and $c_{2}=4$ ), so that $c_{1} \operatorname{diam}\left(Q_{k}\right) \leq \operatorname{distance}\left(Q_{k}, F\right) \leq$ $c_{2} \operatorname{diam}\left(Q_{k}\right)$. Thus, the definition of the homotopy operator $T$ can be generalized to any domain $\Omega$ in $\mathbb{R}^{n}$ : For any $x \in \Omega, x \in Q_{k}$ for some $k$. Let $T_{Q_{k}}$ be the homotopy operator defined on $Q_{k}$ (each cube is bounded and convex). Thus, we can define the homotopy operator $T_{\Omega}$ on any domain $\Omega$ by $T_{\Omega}=\sum_{k=1}^{\infty} T_{Q_{k}} \chi_{Q_{k}}(x)$. The nonlinear partial differential equation for differential forms

$$
d^{\star} A(x, d u)=B(x, d u)
$$

is called a non-homogeneous $A$-harmonic equation, where $A: \Omega \times \wedge^{l}\left(\mathbb{R}^{n}\right) \rightarrow \wedge^{l}\left(\mathbb{R}^{n}\right)$ and $B: \Omega \times \wedge^{l}\left(\mathbb{R}^{n}\right) \rightarrow \wedge^{l-1}\left(\mathbb{R}^{n}\right)$ satisfy the conditions:

$$
|A(x, \xi)| \leq a|\xi|^{p-1}, \quad A(x, \xi) \cdot \xi \geq|\xi|^{p} \quad \text { and } \quad|B(x, \xi)| \leq b|\xi|^{p-1}
$$

for $x \in \Omega$ a.e. and all $\xi \in \wedge^{l}\left(\mathbb{R}^{n}\right)$. Here $p>1$ is a constant related to the equation (1.4), and $a, b>0$. See [11-17] for recent results on the $A$-harmonic equations and related topics. Assume that $\wedge^{l} \Omega$ is the $l$ th exterior power of the cotangent bundle, $C^{\infty}\left(\wedge^{l} \Omega\right)$ is the space of smooth $l$-forms on $\Omega$ and $\mathcal{W}\left(\wedge^{l} \Omega\right)=\left\{u \in L_{\text {loc }}^{1}\left(\wedge^{l} \Omega\right): u\right.$ has generalized gradient $\}$. The harmonic $l$-fields are defined by $\mathcal{H}\left(\wedge^{l} \Omega\right)=\left\{u \in \mathcal{W}\left(\wedge^{l} \Omega\right): d u=d^{\star} u=0, u \in L^{p}\right.$ for some $1<$ $p<\infty\}$. The orthogonal complement of $\mathcal{H}$ in $L^{1}$ is defined by $\mathcal{H}^{\perp}=\left\{u \in L^{1}:\langle u, h\rangle=\right.$ 0 for all $h \in \mathcal{H}\}$. Then the Green's operator $G$ is defined as $G: C^{\infty}\left(\wedge^{l} \Omega\right) \rightarrow \mathcal{H}^{\perp} \cap C^{\infty}\left(\wedge^{l} \Omega\right)$ 
by assigning $G(u)$ be the unique element of $\mathcal{H}^{\perp} \cap C^{\infty}\left(\wedge^{l} \Omega\right)$ satisfying Poisson's equation $\Delta G(u)=u-H(u)$, where $H$ is the harmonic projection operator that maps $C^{\infty}\left(\wedge^{l} \Omega\right)$ onto $\mathcal{H}$ so that $H(u)$ is the harmonic part of $u$. See [18] for more properties of these operators.

\section{Local embedding theorem}

The purpose of this section is to prove the local $L^{\varphi}$-embedding theorem and some related $L^{\varphi}$-norm inequalities that will be used to prove the global embedding theorem in the next section. We first recall the following subclass of Young functions that can be found in [19-21].

Definition 2.1 A Young function $\varphi:[0, \infty) \longrightarrow[0, \infty)$ is said to be in the class $N G(p, q)$ if $\varphi$ satisfies the nonstandard growth condition

$$
p \varphi(t) \leq t \varphi^{\prime}(t) \leq q \varphi(t), \quad 1<p \leq q<\infty
$$

The first inequality in (2.1) is equivalent to that $\frac{\varphi(t)}{t^{p}}$ is increasing, and the second inequality in (2.1) is equivalent to $\Delta_{2}$-condition, i.e., for each $t>0, \varphi(2 t) \leq K \varphi(t)$, where $K>1$, and $\frac{\varphi(t)}{t^{q}}$ is decreasing with $t$. Also, condition (2.1) implies that $\varphi(t)$ satisfies

$$
c_{1} t^{p}-c_{2} \leq \varphi(t) \leq c_{3}\left(t^{q}+1\right) .
$$

Particularly, $\varphi(t)=t^{p}$ satisfies (2.1) because of $t \varphi^{\prime}(t)=p \varphi(t)$, and this makes inequalities with the norm $\|\cdot\|_{p}$ become a special case of Theorem 2.5; for more details see [19] and [20].

An Orlicz function is a continuously increasing function $\varphi:[0, \infty) \rightarrow[0, \infty)$ with $\varphi(0)=0$. The Orlicz space $L^{\varphi}(\Omega)$ consists of all measurable functions $f$ on $\Omega$ such that $\int_{\Omega} \varphi\left(\frac{|f|}{\lambda}\right) d x<\infty$ for some $\lambda=\lambda(f)>0 . L^{\varphi}(\Omega)$ is equipped with the nonlinear Luxemburg functional

$$
\|f\|_{L^{\varphi}(\Omega)}=\inf \left\{\lambda>0: \int_{\Omega} \varphi\left(\frac{|f|}{\lambda}\right) d x \leq 1\right\} .
$$

A convex Orlicz function $\varphi$ is often called a Young function. If $\varphi$ is a Young function, then $\|\cdot\|_{L^{\varphi}(\Omega)}$ defines a norm in $L^{\varphi}(\Omega)$, which is called the Luxemburg norm or Orlicz norm. For any subset $E \subset \mathbb{R}^{n}$, we use $W^{1, \varphi}\left(E, \wedge^{l}\right)$ to denote the Orlicz-Sobolev space of $l$-forms which equals $L^{\varphi}\left(E, \wedge^{l}\right) \cap L_{1}^{\varphi}\left(E, \wedge^{l}\right)$ with norm

$$
\|u\|_{W^{1, \varphi}(E)}=\|u\|_{W^{1, \varphi}\left(E, \wedge^{l}\right)}=\operatorname{diam}(E)^{-1}\|u\|_{L^{\varphi}(E)}+\|\nabla u\|_{L^{\varphi}(E)} .
$$

If we choose $\varphi(t)=t^{p}, p>1$ in (2.3), we obtain the usual $L^{p}$-norm for $W^{1, p}\left(E, \wedge^{l}\right)$

$$
\|u\|_{W^{1, p}(E)}=\|u\|_{W^{1, p}(E, \wedge l)}=\operatorname{diam}(E)^{-1}\|u\|_{p, E}+\|\nabla u\|_{p, E} .
$$

Next, we recall some lemmas that will be used in this paper. 
Lemma 2.2 [9] Let $u \in D^{\prime}\left(B, \wedge^{l}\right)$ and $d u \in L^{p}\left(B, \wedge^{l+1}\right)$. Then $u-u_{B} \in L^{\frac{n p}{n-p}}\left(B, \wedge^{l}\right)$, and

$$
\left(\int_{B}\left|u-u_{B}\right|^{\frac{n p}{n-p}} d x\right)^{\frac{n-p}{n p}} \leq c(n, p)\left(\int_{B}|d u|^{p} d x\right)^{\frac{1}{p}}
$$

for $B$ is a ball or cube in $\Omega, l=0,1, \ldots, n-1$ and $1<p<n$.

Lemma 2.3 [19] Suppose $\varphi$ is a continuous function in the class $N G(p, q)$ with $q(n-p)<n p$, $1<p \leq q<\infty$. For any $t>0$, setting

$$
A(t)=\int_{0}^{t}\left(\frac{\varphi\left(s^{1 / q}\right)}{s}\right)^{\frac{n+q}{q}} d s, \quad K(t)=\frac{\left(\varphi\left(t^{1 / q}\right)\right)^{\frac{n+q}{q}}}{t^{n / q}} .
$$

Then $A(t)$ is a concave function, and there exists a constant $C$, such that

$$
K(t) \leq A(t) \leq C K(t), \quad \forall t>0
$$

Lemma 2.4 [1] Let $u$ be a differential form satisfying the non-homogeneous A-harmonic equation (1.4) in $\Omega, \sigma>1$ and $0<s, t<\infty$. Then, there exists a constant $C$, independent of $u$, such that $\|u\|_{s, B} \leq C|B|^{(t-s) / s t}\|u\|_{t, \sigma B}$ for all balls or cubes $B$ with $\sigma B \subset \Omega$.

We are ready to state our main local $L^{\varphi}$-embedding theorem as follows, which will be used to prove the global $L^{\varphi}$-embedding theorem in the next section.

Theorem 2.5 Let $\varphi$ be a Young function in the class $N G(p, q)$ with $q(n-p)<n p, 1<$ $p \leq q<\infty$. $\Omega$ be a bounded domain, $u \in L^{p}\left(\Omega, \wedge^{l}\right)$ be a solution of the non-homogeneous A-harmonic equation, $T$ be the homotopy operator and $H$ be the projection operator. If $\varphi(|u|) \in L_{\mathrm{loc}}^{1}(\Omega)$, then there exists a constant $C$, independent of $u$, such that

$$
\left\|T(H(u))-(T(H(u)))_{B}\right\|_{W^{1, \varphi}(B, \wedge l)} \leq C|B|\|u\|_{L^{\varphi}(\sigma B)}
$$

for all balls $B$ with $\sigma B \subset \Omega$, where $\sigma>1$ is a constant.

In order to prove the above local $L^{\varphi}$-embedding theorem, we need to prove some local $L^{\varphi}$-norm inequalities. We begin with the following Poincaré-type inequality with $L^{\varphi}$-norm first.

Theorem 2.6 Let $\varphi$ be a Young function in the class $N G(p, q)$ with $q(n-p)<n p, 1<p \leq$ $q<\infty, \Omega$ be a bounded domain, $u \in L^{p}\left(\Omega, \wedge^{l}\right), T$ be the homotopy operator and $H$ be the projection operator. If $\varphi(|u|) \in L_{\mathrm{loc}}^{1}(\Omega)$, then there exists a constant $C$, independent of $u$, such that

$$
\left\|T(H(u))-(T(H(u)))_{B}\right\|_{L^{\varphi}(B)} \leq C|B|\|u\|_{L^{\varphi}(B)}
$$

for all balls $B \subset \Omega$. 
Proof First, we consider the case $1<p<n$. By assumption, we have $q<\frac{n p}{n-p}$. Using the Poincaré-type inequality, Lemma 2.2 to differential forms $T(H(u))$

$$
\left(\int_{B}\left|T(H(u))-(T(H(u)))_{B}\right|^{n p /(n-p)} d x\right)^{(n-p) / n p} \leq C_{1}\left(\int_{B}|d(T(H(u)))|^{p} d x\right)^{1 / p}
$$

we find that

$$
\left(\int_{B}\left|T(H(u))-(T(H(u)))_{B}\right|^{q} d x\right)^{1 / q} \leq C_{2}\left(\int_{B}|d(T(H(u)))|^{p} d x\right)^{1 / p} .
$$

It is well known that, for any differential form $u, d(T(u))=u_{B}$ and $\left\|u_{B}\right\|_{p, B} \leq C_{3}\|u\|_{p, B}$. Hence,

$$
\left(\int_{B}|d(T(H(u)))|^{p} d x\right)^{1 / p} \leq C_{4}\left(\int_{B}|H(u)|^{p} d x\right)^{1 / p} .
$$

Note that

$$
\|\Delta G(u)\|_{p, B}=\left\|\left(d^{\star} d+d d^{\star}\right) G(u)\right\|_{p, B} \leq C_{5}\|u\|_{p, B} .
$$

We have

$$
\begin{aligned}
\|H(u)\|_{p, B} & =\|u-\Delta G(u)\|_{p, B} \\
& \leq\|u\|_{p, B}+\|\Delta G(u)\|_{p, B} \\
& \leq C_{6}\|u\|_{p, B} .
\end{aligned}
$$

Combining (2.11), (2.12), and (2.13), we obtain

$$
\left(\int_{B}\left|T(H(u))-(T(H(u)))_{B}\right|^{q} d x\right)^{1 / q} \leq C_{7}\left(\int_{B}|u|^{p} d x\right)^{1 / p}
$$

for $1<p<n$. Next, for the case of $p \geq n$, since the $L^{p}$-norm of $\left|T(H(u))-(T(H(u)))_{B}\right|$ increases with $p$ and $\frac{n p}{n-p} \rightarrow \infty$ as $p \rightarrow n$. Then, there exists $1<p_{0}<n$ such that $q<\frac{n p_{0}}{n-p_{0}}$. Hence, it follows that

$$
\begin{aligned}
& \left(\int_{B}\left|T(H(u))-(T(H(u)))_{B}\right|^{q} d x\right)^{1 / q} \\
& \quad \leq\left(\int_{B}\left|T(H(u))-(T(H(u)))_{B}\right|^{n p_{0} /\left(n-p_{0}\right)} d x\right)^{\left(n-p_{0}\right) / n p_{0}} \\
& \quad \leq C_{8}\left(\int_{B}|d(T(H(u)))|^{p_{0}} d x\right)^{1 / p_{0}} \\
& \quad \leq C_{9}\left(\int_{B}|d(T(H(u)))|^{p} d x\right)^{1 / p} \\
& \quad \leq C_{10}\left(\int_{B}|u|^{p} d x\right)^{1 / p} .
\end{aligned}
$$


Hence, from (2.14) and (2.15), we obtain

$$
\left(\int_{B}\left|T(H(u))-(T(H(u)))_{B}\right|^{q} d x\right)^{1 / q} \leq C_{10}\left(\int_{B}|u|^{p} d x\right)^{1 / p}
$$

for any $p>1$. Using the Hölder inequality with $1=\frac{q}{n+q}+\frac{n}{n+q}$, we obtain

$$
\begin{aligned}
& \int_{B} \varphi\left(\left|T(H(u))-(T(H(u)))_{B}\right|\right) d x \\
& =\int_{B} \frac{\varphi\left(\left|T(H(u))-(T(H(u)))_{B}\right|\right)}{\left|T(H(u))-(T(H(u)))_{B}\right|^{\frac{n q}{n+q}}}\left|T(H(u))-(T(H(u)))_{B}\right|^{\frac{n q}{n+q}} d x \\
& \leq\left(\int_{B} \frac{\varphi\left(\left|T(H(u))-(T(H(u)))_{B}\right|\right)^{\frac{n+q}{q}}}{\left|T(H(u))-(T(H(u)))_{B}\right|^{n}} d x\right)^{\frac{q}{n+q}} \\
& \quad \times\left(\int_{B}\left|T(H(u))-(T(H(u)))_{B}\right|^{q} d x\right)^{\frac{n}{n+q}} .
\end{aligned}
$$

Applying Lemma 2.3 and noticing $A(t)$ is a concave function, we obtain

$$
\begin{aligned}
\int_{B} \varphi & \left(\left|T(H(u))-(T(H(u)))_{B}\right|\right) d x \\
\leq & \left(\int_{B} K\left(\left|T(H(u))-(T(H(u)))_{B}\right|^{q}\right) d x\right)^{\frac{q}{n+q}}\left(\int_{B}\left|T(H(u))-(T(H(u)))_{B}\right|^{q} d x\right)^{\frac{n}{n+q}} \\
\leq & \left(\int_{B} A\left(\left|T(H(u))-(T(H(u)))_{B}\right|^{q}\right) d x\right)^{\frac{q}{n+q}}\left(\int_{B}\left|T(H(u))-(T(H(u)))_{B}\right|^{q} d x\right)^{\frac{n}{n+q}} \\
\leq & A^{\frac{q}{n+q}}\left(\int_{B}\left(\left|T(H(u))-(T(H(u)))_{B}\right|^{q}\right) d x\right)\left(\int_{B}\left|T(H(u))-(T(H(u)))_{B}\right|^{q} d x\right)^{\frac{n}{n+q}} \\
\leq & C_{1}(n, q) K^{\frac{q}{n+q}}\left(\int_{B}\left(\left|T(H(u))-(T(H(u)))_{B}\right|^{q}\right) d x\right) \\
& \times\left(\int_{B}\left|T(H(u))-(T(H(u)))_{B}\right|^{q} d x\right)^{\frac{n}{n+q}} \\
= & C_{1}(n, q) \frac{\varphi\left(\left(\int_{B}\left(\left|T(H(u))-(T(H(u)))_{B}\right|^{q}\right) d x\right)^{1 / q}\right)}{\left(\int_{B}\left(\left|T(H(u))-(T(H(u)))_{B}\right|^{q}\right) d x\right)^{\frac{n}{n+q}}} \\
& \times\left(\int_{B}\left|T(H(u))-(T(H(u)))_{B}\right|^{q} d x\right)^{\frac{n}{n+q}} \\
= & C_{1}(n, q) \varphi\left(\left(\int_{B}\left(\left|T(H(u))-(T(H(u)))_{B}\right|^{q}\right) d x\right)^{1 / q}\right.
\end{aligned} .
$$

Note that $\varphi$ is increasing and satisfies $\Delta_{2}$-condition, substituting (2.16) into (2.17) gives

$$
\int_{B} \varphi\left(\left|T(H(u))-(T(H(u)))_{B}\right|\right) d x \leq C_{11} \varphi\left(\left(\int_{B}|u|^{p} d x\right)^{1 / p}\right) .
$$


Let $h(t)=\int_{0}^{t} \frac{\varphi(s)}{s} d s$. From (2.1) we know that $\varphi(t) / t^{q}$ is decreasing with $t$, thus,

$$
h(t)=\int_{0}^{t} \frac{\varphi(s)}{s} d s=\int_{0}^{t} \frac{\varphi(s)}{s^{q}} s^{q-1} d s \geq \varphi(t) /\left.t^{q} \frac{1}{q} s^{q}\right|_{0} ^{t}=\frac{1}{q} \varphi(t) .
$$

Similarly, using the fact that $\varphi(t) / t^{p}$ is increasing with $t$, we have $h(t) \leq \frac{1}{p} \varphi(t)$. Therefore,

$$
\frac{1}{q} \varphi(t) \leq h(t) \leq \frac{1}{p} \varphi(t)
$$

Let $g(t)=h\left(t^{1 / p}\right)$, then $\left(h\left(t^{1 / p}\right)\right)^{\prime}=\frac{1}{p} \frac{\varphi\left(t^{1 / p}\right)}{t}$ is increasing. Hence, $g$ is a convex function. From the definitions of $g$ and $h$ and using Jensen's inequality to $g$, we have

$$
h\left(\left(\int_{B}|u|^{p} d x\right)^{1 / p}\right)=g\left(\int_{B}|u|^{p} d x\right) \leq \int_{B} g\left(|u|^{p}\right) d x=\int_{B} h(|u|) d x .
$$

Combining (2.18), (2.19), and (2.20), we have

$$
\begin{aligned}
& \int_{B} \varphi\left(\left|T(H(u))-(T(H(u)))_{B}\right|\right) d x \\
& \leq C_{11} \varphi\left(\left(\int_{B}|u|^{p} d x\right)^{1 / p}\right) \\
& \leq C_{12} h\left(\left(\int_{B}|u|^{p} d x\right)^{1 / p}\right) \\
& \leq C_{13} \int_{B} h(|u|) d x \\
& \leq C_{14} \int_{B} \varphi(|u|) d x,
\end{aligned}
$$

which indicates (2.9) holds. We have completed the proof of Theorem 2.6.

Theorem 2.7 Let $\varphi$ be a Young function in the class $N G(p, q)$ with $q(n-p)<n p, 1<$ $p \leq q<\infty, \Omega$ be a bounded domain, $u \in L^{p}\left(\Omega, \wedge^{l}\right)$ be a solution of the non-homogeneous A-harmonic equation, $T$ be the homotopy operator and $H$ be the projection operator. If $\varphi(|u|) \in L_{\text {loc }}^{1}(\Omega)$, then there exists a constant $C$, independent of $u$, such that

$$
\|\operatorname{TdTH}(u)\|_{L^{\varphi}(B)} \leq C|B|\|u\|_{L^{\varphi}(\sigma B)}
$$

for all balls $B$ with $\sigma B \subset \Omega$, where $\sigma>1$ is a constant.

Proof For any differential form $u$, we have $\|d T(u)\|_{q, B}=\left\|u_{B}\right\|_{q, B} \leq C_{1}\|u\|_{q, B}$. From (1.3) and (2.13), it follows that

$$
\begin{aligned}
\|\operatorname{TdTH}(u)\|_{q, B} & \leq C_{2}|B| \operatorname{diam}(B)\|d T H(u)\|_{q, B} \\
& =C_{2}|B| \operatorname{diam}(B)\|H(u)\|_{q, B} \\
& =C_{3}|B| \operatorname{diam}(B)\|u\|_{q, B} .
\end{aligned}
$$


By Lemma 2.4 and $p, q>0$, we have

$$
\|u\|_{q, B} \leq C_{4}|B|^{(p-q) / p q}\|u\|_{p, \sigma B}
$$

where $\sigma>1$. Combining (2.21) and (2.22) yields

$$
\|\operatorname{Td} T H(u)\|_{q, B} \leq C_{5}|B|^{(p-q) / p q}\|u\|_{p, \sigma B} .
$$

From the Hölder inequality with $1=\frac{q}{n+q}+\frac{n}{n+q}$, we find that

$$
\begin{aligned}
& \int_{B} \varphi(|\operatorname{TdTH}(u)|) d x \\
& \quad=\int_{B} \varphi(|\operatorname{TdTH}(u)|)(|\operatorname{TdTH}(u)|)^{-\frac{n q}{n+q}}(|\operatorname{TdTH}(u)|)^{\frac{n q}{n+q}} d x \\
& \quad \leq\left(\int_{B} \frac{\varphi(|\operatorname{TdTH}(u)|)^{\frac{n+q}{q}}}{|\operatorname{TdTH}(u)|^{n}} d x\right)^{\frac{q}{n+q}}\left(\int_{B}|\operatorname{TdTH}(u)|^{q} d x\right)^{\frac{n}{n+q}} .
\end{aligned}
$$

Using Lemma 2.3 and noticing $A(t)$ is a concave function, we obtain

$$
\begin{aligned}
& \int_{B} \varphi(|\operatorname{TdTH}(u)|) d x \\
& \leq\left(\int_{B} K\left(|\operatorname{TdTH}(u)|^{q}\right) d x\right)^{\frac{q}{n+q}}\left(\int_{B}|\operatorname{TdTH}(u)|^{q} d x\right)^{\frac{n}{n+q}} \\
& \leq\left(\int_{B} A\left(|\operatorname{TdTH}(u)|^{q}\right) d x\right)^{\frac{q}{n+q}}\left(\int_{B}|\operatorname{TdTH}(u)|^{q} d x\right)^{\frac{n}{n+q}} \\
& \leq A^{\frac{q}{n+q}}\left(\int_{B}\left(|\operatorname{TdTH}(u)|^{q}\right) d x\right)\left(\int_{B}|\operatorname{TdTH}(u)|^{q} d x\right)^{\frac{n}{n+q}} \\
& \leq C_{6} K^{\frac{q}{n+q}}\left(\int\left(|\operatorname{TdTH}(u)|^{q}\right) d x\right)\left(\int_{B}|\operatorname{TdTH}(u)|^{q} d x\right)^{\frac{n}{n+q}} \\
& \quad=C_{6} \frac{\varphi\left(\left(\int_{B}\left(|\operatorname{TdTH}(u)|^{q}\right) d x\right)^{1 / q}\right)}{\left(\int_{B}\left(|\operatorname{TdTH}(u)|^{q}\right) d x\right)^{\frac{n}{n+q}}}\left(\int_{B}|\operatorname{TdTH}(u)|^{q} d x\right)^{\frac{n}{n+q}} \\
& \quad=C_{6} \varphi\left(\left(\int_{B}\left(|\operatorname{TdTH}(u)|^{q}\right) d x\right)^{1 / q}\right) .
\end{aligned}
$$

Since $\varphi$ is increasing and satisfies $\Delta_{2}$-condition, substituting (2.23) into (2.24), we have

$$
\int_{B} \varphi(|\operatorname{TdTH}(u)|) d x \leq C_{7} \varphi\left(\left(\int_{\sigma B}|u|^{p} d x\right)^{1 / p}\right) .
$$

Starting from (2.25) and using the same discussion as we did in the proof of Theorem 2.6, we obtain

$$
\int_{B} \varphi(|\operatorname{TdTH}(u)|) d x \leq C \int_{\sigma B} \varphi(|u|) d x
$$

We have completed the proof of Theorem 2.7. 
From (1.3) and (2.13), we have

$$
\begin{aligned}
\|\nabla \operatorname{TdTH}(u)\|_{q, B} & \leq C_{1}|B|\|d T H(u)\|_{q, B} \\
& =C_{1}|B|\left\|(H(u))_{B}\right\|_{q, B} \\
& \leq C_{2}|B|\|H(u)\|_{q, B} \\
& \leq C_{3}|B|\|u\|_{q, B} .
\end{aligned}
$$

Using (2.26) and the similar techniques to the ones developed in the proof of Theorem 2.7, we obtain the following $L^{\varphi}$-norm estimate.

Theorem 2.8 Let $\varphi$ be a Young function in the class $N G(p, q)$ with $q(n-p)<n p, 1<p \leq$ $q<\infty, \Omega$ be a bounded domain, and $u \in L^{p}\left(\Omega, \wedge^{l}\right)$ be a solution of the non-homogeneous A-harmonic equation, $T$ be the homotopy operator and $H$ be the projection operator. If $\varphi(|u|) \in L_{\mathrm{loc}}^{1}(\Omega)$, then there exists a constant $C$, independent of $u$, such that

$$
\|\nabla \operatorname{TdTH}(u)\|_{L^{\varphi}(B)} \leq C|B|\|u\|_{L^{\varphi}(\sigma B)}
$$

for all balls $B$ with $\sigma B \subset \Omega$, where $\sigma>1$ is a constant.

Proof of Theorem 2.5 By definition (2.3), Theorem 2.7 and Theorem 2.8, we have

$$
\begin{aligned}
& \left\|T(H(u))-(T(H(u)))_{B}\right\|_{W^{1, \varphi}\left(B, \wedge^{l}\right)} \\
& \quad=\|\operatorname{TdTH}(u)\|_{W^{1, \varphi}\left(B, \wedge^{l}\right)} \\
& \quad=\operatorname{diam}(B)^{-1}\|\operatorname{TdTH}(u)\|_{L^{\varphi}(B)}+\|\nabla T d T H(u)\|_{L^{\varphi}(B)} \\
& \quad \leq \operatorname{diam}(B)^{-1} C_{1}|B| \operatorname{diam}(B)\|u\|_{L^{\varphi}\left(\sigma_{1} B\right)}+C_{2}|B|\|u\|_{L^{\varphi}\left(\sigma_{2} B\right)} \\
& \quad \leq C_{3}|B|\|u\|_{\left.L^{\varphi}(\sigma B)\right)}
\end{aligned}
$$

where $\sigma=\max \left\{\sigma_{1}, \sigma_{2}\right\}$. We have completed the proof of Theorem 2.5.

\section{Global embedding theorem}

In this section, we prove our main result, the global $L^{\varphi}$-embedding theorem for the solutions of the non-homogeneous $A$-harmonic equation. We will use the following wellknown covering lemma.

Lemma 3.1 Each domain $\Omega$ has a modified Whitney cover of cubes $\mathcal{V}=\left\{Q_{i}\right\}$ such that

$$
\bigcup_{i} Q_{i}=\Omega, \quad \sum_{Q_{i} \in \mathcal{V}} \chi_{\sqrt{\frac{5}{4}} Q_{i}} \leq N \chi_{\Omega}
$$

and some $N>1$, and if $Q_{i} \cap Q_{j} \neq \emptyset$, then there exists a cube $R$ (this cube need not be a member of $\mathcal{V}$ ) in $Q_{i} \cap Q_{j}$ such that $Q_{i} \cup Q_{j} \subset N R$. Moreover, if $\Omega$ is $\delta$-John, then there is a distinguished cube $Q_{0} \in \mathcal{V}$ which can be connected with every cube $Q \in \mathcal{V}$ by a chain of cubes $Q_{0}, Q_{1}, \ldots, Q_{k}=Q$ from $\mathcal{V}$ and such that $Q \subset \rho Q_{i}, i=0,1,2, \ldots, k$, for some $\rho=\rho(n, \delta)$. 
We are ready to prove the following global $L^{\varphi}$-embedding theorem with the $L^{\varphi}$-norm now.

Theorem 3.2 Let $\varphi$ be a Young function in the class $N G(p, q)$ with $q(n-p)<n p, 1<p \leq$ $q<\infty, u \in L^{p}\left(\Omega, \wedge^{l}\right)$ be a solution of the non-homogeneous $A$-harmonic equation, $T$ be the homotopy operator and $H$ be the projection operator. If $\varphi(|u|) \in L^{1}(\Omega)$, then there exists a constant $C$, independent of $u$, such that

$$
\left\|T(H(u))-(T(H(u)))_{\Omega}\right\|_{W^{1, \varphi}(\Omega, \wedge l)} \leq C\|u\|_{L^{\varphi}(\Omega)}
$$

for a bounded domain $\Omega \subset \mathbb{R}^{n}$.

Proof From the Lemma 3.1 and Theorem 2.8, we have

$$
\begin{aligned}
\|\nabla T d(T(H(u)))\|_{L^{\varphi}(\Omega)} & \leq \sum_{B \in \mathcal{V}}\|\nabla T d(T(H(u)))\|_{L^{\varphi}(B)} \\
& \leq \sum_{B \in \mathcal{V}}\left(C_{1}|B|\|u\|_{L^{\varphi}(\sigma B)}\right) \\
& \leq C_{2} N\|u\|_{L^{\varphi}(\Omega)} \\
& \leq C_{3}\|u\|_{L^{\varphi}(\Omega)} .
\end{aligned}
$$

Similarly, from Theorem 2.7 and Lemma 3.1, it follows that

$$
\begin{aligned}
\|T d(T(H(u)))\|_{L^{\varphi}(\Omega)} & \leq \sum_{B \in \mathcal{V}}\|T d(T(H(u)))\|_{L^{\varphi}(B)} \\
& \leq \sum_{B \in \mathcal{V}}\left(C_{4} \operatorname{diam}(B)\|u\|_{L^{\varphi}(\sigma B)}\right) \\
& \leq C_{5} \operatorname{diam}(\Omega) N\|u\|_{L^{\varphi}(\Omega)} \\
& \leq C_{6} \operatorname{diam}(\Omega)\|u\|_{L^{\varphi}(\Omega)}
\end{aligned}
$$

Using (2.3), (3.2), and (3.3), we find that

$$
\begin{aligned}
& \left\|T(H(u))-(T(H(u)))_{\Omega}\right\|_{W^{1, \varphi}(\Omega)} \\
& \quad=\|\operatorname{Td}(T(H(u)))\|_{W^{1, \varphi}(\Omega)} \\
& \quad=(\operatorname{diam}(\Omega))^{-1}\|T d(T(H(u)))\|_{L^{\varphi}(\Omega)}+\|\nabla T d(T(H(u)))\|_{L^{\varphi}(\Omega)} \\
& \quad \leq(\operatorname{diam}(\Omega))^{-1}\left(C_{6} \operatorname{diam}(\Omega)\|u\|_{L^{\varphi}(\Omega)}\right)+C_{3}\|u\|_{L^{\varphi}(\Omega)} \\
& \quad \leq C_{7}\|u\|_{L^{\varphi}(\Omega)} .
\end{aligned}
$$

We have completed the proof of Theorem 3.2.

Choosing $\varphi(t)=t^{p} \log _{+}^{\alpha} t$ in Theorems 3.2, we have the following embedding inequality with the $L^{p}\left(\log _{+}^{\alpha} L\right)$-norms. 
Corollary 3.3 Let $\varphi(t)=t^{p} \log _{+}^{\alpha} t, p \geq 1, \alpha \in \mathbb{R}, u \in L^{p}\left(\Omega, \wedge^{l}\right)$ be a solution of the nonhomogeneous A-harmonic equation, $T$ be the homotopy operator, and $H$ be the projection operator. If $\varphi(|u|) \in L^{1}(\Omega)$, then there exists a constant $C$, independent of $u$, such that

$$
\left\|T(H(u))-(T(H(u)))_{\Omega}\right\|_{W^{1, t^{p}} \log _{+}^{\alpha} t_{(\Omega)}} \leq C\|u\|_{L^{t p} \log _{+}^{\alpha} t(\Omega)}
$$

for any bounded domain $\Omega$.

Let $\varphi(t)=t^{p}$ in Theorem 3.2. Then, we obtain the following version of the embedding inequality with $L^{p}$-norms.

Corollary 3.4 Let $\varphi(t)=t^{p}, p \geq 1, u \in L^{p}\left(\Omega, \wedge^{l}\right)$ be a solution of the non-homogeneous A-harmonic equation, $T$ be the homotopy operator, and $H$ be the projection operator. If $\varphi(|u|) \in L^{1}(\Omega)$, then there exists a constant $C$, independent of $u$, such that

$$
\left\|T(H(u))-(T(H(u)))_{\Omega}\right\|_{W^{1, p}(\Omega)} \leq C\|u\|_{p, \Omega}
$$

holds for any bounded domain $\Omega$.

Similarly, Theorem 2.6 can be extended into the following global Poincaré-type inequality with $L^{\varphi}$-norm.

Theorem 3.5 Let $\varphi$ be a Young function in the class $N G(p, q)$ with $q(n-p)<n p, 1<p \leq$ $q<\infty, u \in L^{p}\left(\Omega, \wedge^{l}\right)$ be a solution of the non-homogeneous $A$-harmonic equation, $T$ be the homotopy operator and $H$ be the projection operator. If $\varphi(|u|) \in L^{1}(\Omega)$, then there exists a constant $C$, independent of $u$, such that

$$
\left\|T(H(u))-(T(H(u)))_{\Omega}\right\|_{L^{\varphi}(\Omega)} \leq C \operatorname{diam}(\Omega)\|u\|_{L^{\varphi}(\Omega)}
$$

for any bounded domain $\Omega$.

Proof From (2.3) and Theorem 3.2, we have

$$
\begin{aligned}
\left\|T(H(u))-(T(H(u)))_{\Omega}\right\|_{L^{\varphi}(\Omega)} \\
=\operatorname{diam}(\Omega)\left((\operatorname{diam}(\Omega))^{-1}\left\|T(H(u))-(T(H(u)))_{\Omega}\right\|_{L^{\varphi}(\Omega)}\right) \\
\leq \operatorname{diam}(\Omega)\left((\operatorname{diam}(\Omega))^{-1}\left\|T(H(u))-(T(H(u)))_{\Omega}\right\|_{L^{\varphi}(\Omega)}\right. \\
\left.\quad+\left\|\nabla\left(T(H(u))-(T(H(u)))_{\Omega}\right)\right\|_{L^{\varphi}(\Omega)}\right) \\
=C_{1} \operatorname{diam}(\Omega)\left\|T(H(u))-(T(H(u)))_{\Omega}\right\|_{W^{1, \varphi}(\Omega, \wedge l} \\
\leq C_{2} \operatorname{diam}(\Omega)\|u\|_{L^{\varphi}(\Omega) .}
\end{aligned}
$$

We have completed the proof of Theorem 3.5. 


\section{Applications}

As applications of our main results established in the previous sections, we consider the following examples.

Example 4.1 Assume that $r>0$ and $k>0$ are any constants and $\Omega=\left\{\left(x_{1}, x_{2}, x_{3}\right): x_{1}^{2}+x_{2}^{2}+\right.$ $\left.x_{3}^{2} \leq r^{2}\right\} \subset \mathbb{R}^{3}$. Consider the 1 -form

$$
u\left(x_{1}, x_{2}, x_{3}\right)=\frac{x_{1}}{k+x_{1}^{2}+x_{2}^{2}+x_{3}^{2}} d x_{1}+\frac{x_{2}}{k+x_{1}^{2}+x_{2}^{2}+x_{3}^{2}} d x_{2}+\frac{x_{3}}{k+x_{1}^{2}+x_{2}^{2}+x_{3}^{2}} d x_{3}
$$

defined in $\Omega$. It is easy to check that $d u=0$. Hence, $u$ is a solution of the non-homogeneous $A$-harmonic equation (1.4) for any operators $A$ and $B$ satisfying (1.5). Also, it can be calculated that

$$
\begin{aligned}
|u| & =\left(\left(\frac{x_{1}}{k+x_{1}^{2}+x_{2}^{2}+x_{3}^{2}}\right)^{2}+\left(\frac{x_{2}}{k+x_{1}^{2}+x_{2}^{2}+x_{3}^{2}}\right)^{2}+\left(\frac{x_{3}}{k+x_{1}^{2}+x_{2}^{2}+x_{3}^{2}}\right)^{2}\right)^{1 / 2} \\
& =\left(\frac{x_{1}^{2}+x_{2}^{2}+x_{3}^{2}}{\left(k+x_{1}^{2}+x_{2}^{2}+x_{3}^{2}\right)^{2}}\right)^{1 / 2}<1 .
\end{aligned}
$$

Using (4.2) and (3.1), we find that

$$
\left\|T(H(u))-(T(H(u)))_{\Omega}\right\|_{W^{1, \varphi}(\Omega)} \leq C_{1}\|u\|_{L^{\varphi}(\Omega)} \leq C_{2}\|1\|_{L^{\varphi}(\Omega)},
$$

that is,

$$
\left\|T(H(u))-(T(H(u)))_{\Omega}\right\|_{W^{1, \varphi}(\Omega)} \leq C_{2} \int_{\Omega} \varphi\left(\frac{1}{\lambda}\right) d x \leq C_{3} r^{3} .
$$

We should notice that the above example can be extended to the case of $\mathbb{R}^{n}$. Specifically, we can check that the 1 -form defined in $\mathbb{R}^{n}$

$$
u\left(x_{1}, \ldots, x_{n}\right)=\sum_{i=1}^{n} \frac{x_{i}}{k+x_{1}^{2}+\cdots+x_{n}^{2}} d x_{i}, \quad k>0
$$

is a solution of the non-homogeneous $A$-harmonic equation (1.4) for any operators $A$ and $B$ satisfying (1.5). Hence, Theorem 3.2 is applicable to $u\left(x_{1}, \ldots, x_{n}\right)$ as we did in Example 4.1. Finally, we consider the following example in $\mathbb{R}^{3}$.

Example 4.2 Let $\Omega=\left\{(x, y, z): x^{2}+y^{2}+z^{2} \leq 1\right\} \subset \mathbb{R}^{3}$ and $u(x, y, z)$ be defined in $\mathbb{R}^{3}$ by

$$
u(x, y, z)=e^{x^{2}+y^{2}+z^{2}}(x d x+y d y+z d z) .
$$

It is easy to check that $d u=0$. Hence, $u$ is a solution of the non-homogeneous $A$-harmonic equation (1.4) for any operators $A$ and $B$ satisfying (1.5) in $\mathbb{R}^{3}$. For any bounded domain $\Omega$ in $\mathbb{R}^{3}$, it would be very complicated if we calculate the integral $\| T(H(u))$ $(T(H(u)))_{\Omega} \|_{W^{1, \varphi}(\Omega)}$ directly. However, using the embedding inequality (3.1), we can easily obtain the upper bound of the norm $\left\|T(H(u))-(T(H(u)))_{\Omega}\right\|_{W^{1, \varphi}(\Omega)}$ as follows. We notice 
that

$$
\begin{aligned}
|u(x, y, z)| & =\left(\left(e^{x^{2}+y^{2}+z^{2}} x\right)^{2}+\left(e^{x^{2}+y^{2}+z^{2}} y\right)^{2}+\left(e^{x^{2}+y^{2}+z^{2}} z\right)^{2}\right)^{1 / 2} \\
& =\left(e^{2\left(x^{2}+y^{2}+z^{2}\right)}\left(x^{2}+y^{2}+z^{2}\right)\right)^{1 / 2} \\
& \leq\left(e^{2} \cdot 1\right)^{1 / 2}=e
\end{aligned}
$$

From (4.4) and (3.1), we have

$$
\left\|T(H(u))-(T(H(u)))_{\Omega}\right\|_{W^{1, \varphi}(\Omega)} \leq C_{1}\|u\|_{L^{\varphi}(\Omega)} \leq C_{2}\|e\|_{L^{\varphi}(\Omega)},
$$

which is equal to

$$
\left\|T(H(u))-(T(H(u)))_{\Omega}\right\|_{W^{1, \varphi}(\Omega)} \leq C_{2} \int_{\Omega} \varphi\left(\frac{e}{\lambda}\right) d x \leq C_{3} .
$$

Remark (i) From inequalities (3.1), (3.2), and (3.3), we find that the compositions $T H$, $T d T H$ and $\nabla T d T H$ are bounded operators on $L^{p}\left(\Omega, \wedge^{l}\right)$. (ii) Note that our global embedding theorem holds on any bounded domains. Hence, the theorem is true if $\Omega$ is one of the bounded John domains, $L^{p}$-averaging domains or $L^{\varphi}(\mu)$-averaging domains. See [1, 22,23 ] for more properties of these kinds of domains.

\section{Competing interests}

The authors declare that there is no conflict of interests regarding the publication of this article.

\section{Authors' contributions}

All authors worked together on the research and writing of this manuscript. JN carried out the proofs of all research results in this manuscript, and wrote its draft. SD and YX proposed the study, participated in its design and revised its final version. All authors read and approved the final manuscript.

\section{Author details}

${ }^{1}$ Department of Mathematics, Harbin Institute of Technology, Harbin, 150001, China. ${ }^{2}$ Department of Mathematics, Seattle University, Seattle, WA 98122, USA.

Received: 14 August 2015 Accepted: 1 November 2015 Published online: 02 December 2015

\section{References}

1. Agarwal, RP, Ding, S, Nolder, CA: Inequalities for Differential Forms. Springer, Berlin (2009)

2. Xing, Y, Wu, C: Global weighted inequalities for operators and harmonic forms on manifolds. J. Math. Anal. Appl. 294, 294-309 (2004)

3. $\mathrm{Bi}, \mathrm{H}$ : Weighted inequalities for potential operators on differential forms. J. Inequal. Appl. (2010). doi:10.1155/2010/713625

4. $\mathrm{Bi}, \mathrm{H}$, Ding, S: Some strong $(p, q)$-type inequalities for the homotopy operator. Comput. Math. Appl. 62, 1780-1789 (2011)

5. Ding, S, Liu, B: A singular integral of the composite operator. Appl. Math. Lett. 22, 1271-1275 (2009)

6. Ding, S, Liu, B: Global estimates for singular integrals of the composite operator. III. J. Math. 53, 1173-1185 (2009)

7. Bi, H, Ding, S: Orlicz norm inequalities for the composite operator and applications. J. Inequal. Appl. (2011). doi:10.1186/1029-242X-2011-69

8. Cartan, H: Differential Forms. Houghton Mifflin, Boston (1970)

9. Iwaniec, T, Lutoborski, A: Integral estimates for null Lagrangians. Arch. Ration. Mech. Anal. 125, 25-79 (1993)

10. Stein, EM: Harmonic Analysis. Princeton University Press, Princeton (1993)

11. Ding, S: Two-weight Caccioppoli inequalities for solutions of nonhomogeneous A-harmonic equations on Riemannian manifolds. Proc. Am. Math. Soc. 132, 2367-2375 (2004)

12. Ding, S, Liu, B: Norm inequalities for composition of Dirac and Green's operators. J. Inequal. Appl. (2013). doi:10.1186/1029-242x-2013-436

13. Wang, Y, Wu, C: Sobolev embedding theorems and Poincaré inequalities for Green's operator on solutions of the nonhomogeneous A-harmonic equation. Comput. Math. Appl. 47, 1545-1554 (2004)

14. Liu, B: $A_{f}^{\lambda}(\Omega)$-Weighted imbedding inequalities for A-harmonic tensors. J. Math. Anal. Appl. 273, $667-676$ (2002)

15. Wang, $Y$ : Two-weight Poincaré type inequalities for differential forms in $L^{5}(\mu)$-averaging domains. Appl. Math. Lett. 20,1161-1166 (2007) 
16. Xing, Y: Weighted Poincaré-type estimates for conjugate A-harmonic tensors. J. Inequal. Appl. (2005). doi:10.1155/JAA.2005.1

17. Xing, Y: Weighted integral inequalities for solutions of the A-harmonic equation. J. Math. Anal. Appl. 279, 350-363 (2003)

18. Scott, C: $L^{p}$-Theory of differential forms on manifolds. Trans. Am. Math. Soc. 347, 2075-2096 (1995)

19. Fusco, N, Sbordone, C: Higher integrability of the gradient of minimizers of functionals with nonstandard growth conditions. Commun. Pure Appl. Math. 43, 673-683 (1990)

20. Sbordone, C: Maximal inequalities and applications to regularity problems in the calculus of variations. Pitman Res. Notes Math. Ser. 326, 230-244 (1995)

21. $L u, Y, B a o, G$ : Poincaré inequality and the sharp maximal inequalities with $L^{\varphi}$-norms for differential forms. J. Inequal. Appl. (2013). doi:10.1186/1029-242X-2013-400

22. Staples, SG: LP-Averaging domains and the Poincaré inequality. Ann. Acad. Sci. Fenn., Ser. A 1 Math. 14, 103-127 (1989)

23. Ding, $S: L^{\varphi}(\mu)$-Averaging domains and the quasihyperbolic metric. Comput. Math. Appl. 47, 1611-1618 (2004)

Submit your manuscript to a SpringerOpen ${ }^{\circ}$ journal and benefit from:

- Convenient online submission

- Rigorous peer review

- Immediate publication on acceptance

Open access: articles freely available online

- High visibility within the field

- Retaining the copyright to your article 